\title{
Assessing the social impact of direct-to-consumer genetic testing: Understanding sociotechnical architectures
}

\author{
Shobita Parthasarathy, $P h D$
}

\begin{abstract}
To properly understand the social impact of direct-to-consumer genetic testing, we must consider the "sociotechnical architectures" of these technologies - how developers design and assemble the human and technical components of individual testing systems to perform specific functions. In particular, the way testing systems perform their main functions - providing access to testing, analyzing genetic material, and conveying test results - influence the technology's utility and the distribution of expertise in the medical system. I illustrate this concept by comparing two systems that offer single-nucleotide polymorphism analysis, a relatively new type of genetic testing. I conclude by exploring how policy officials and other decision makers might intervene in the development of sociotechnical architectures to maximize the benefits of genomic technologies. Genet Med 2010:12(9):544-547.
\end{abstract}

Key Words: genetic testing, policy, personalized medicine, utility, ethics

G enetic testing technologies could have a significant impact on contemporary societies. In recent years, policymakers, public health officials, physicians, and scientists have devoted significant attention to the potential implications of these innovations, to determine whether and how they should be regulated. ${ }^{1-3}$ These analyses often focus on either the technical accuracy of these tests or the broad impact of this whole class of technologies. ${ }^{4-6}$ In this commentary, I argue that to properly anticipate social impact and develop appropriate policies in this area, we must think carefully about technological design. To facilitate this kind of assessment, I discuss the concept of "sociotechnical architectures"- the human and technical components of innovations and the way developers fit them together to perform specific functions. Identifying sociotechnical architectures helps us illuminate how the choice of each component and their assembly into a functioning whole influences a technology's social consequences. This concept, which I have discussed in more detail elsewhere, ${ }^{7}$ is based on research from the field of science and technology studies, which has demonstrated that the way that a technology is not only marketed but also built, shapes its social implications. ${ }^{8-11}$

Just as a building's architecture is the orderly organization of materials and components to achieve a functional, economical, and practical building, a technology's "sociotechnical architec-

From the Ford School of Public Policy, University of Michigan, Ann Arbor, Michigan.

Shobita Parthasarathy, PhD, Ford School of Public Policy, University of Michigan, 4202 Weill Hall, 735 S. State Street, Ann Arbor, MI 48109-3091. E-mail: shobita@umich.edu.

Disclosure: The author declares no conflict of interest

Submitted for publication December 22, 2009

Accepted for publication May 7, 2010.

Published online ahead of print July 1, 2010.

DOI: $10.1097 /$ GIM.0b013e3181e71c70 ture" incorporates functional, social, and ethical considerations to serve human needs. Thus, to conduct an architectural assessment, we must first identify the functions of the technological system being assessed. We then identify the human and technical components that fulfill those functions - how the technology is packaged, how it is offered, inherent safeguards and limitations in the offering, and its cost. Finally, we study how the choice, design, and assembly of these components "structure" the technology's implications, by influencing the way system participants interact with, and think and make decisions about, the technology. Similar to a building's architecture that limits who can enter and how they move inside, the architecture of a genetic testing system influences a client's right to privacy, understanding of familial relationships, and the health care professional's level of authority, among other things.

Testing for BRCA1 and BRCA2 genes, for example, is designed to assess a client's genetic predisposition for breast and ovarian cancer. Genetic testing systems perform three main functions: providing access to the test, analyzing biological material, and conveying test results. Access could be provided directly to the user or with the guidance of a health care professional. Providers might analyze biological material by fully sequencing genes or by targeting areas where diseasecausing mutations are most likely to occur. Test results might be conveyed to users through email, telephone, during an appointment with a physician, or in a group meeting with an interdisciplinary team of concerned professionals. The design and assembly of components structures the effectiveness and autonomy of the participants involved in the technological system-clients, health care professionals, and test providers - in specific ways. The different ways of accessing BRCA testing in the United States and Britain have different effects. In the United States, clients can purchase the test through any physician, signifying an empowered consumer who has the right to access testing on demand. However, the client is responsible for the cost and for dealing with the resulting complicated information about her cancer risk essentially independently. By contrast, the British test is made available only to prescreened "high-risk" individuals who have undergone genetic counseling. Here, the client is a traditional patient who benefits from the guidance and gatekeeping of medical professionals, albeit at the expense of her independence and privacy. In this example, we see that the differences in the architecture of the testing technology directly influence the doctor-patient relationship and the distribution of expertise in the medical system in different ways.

The remainder of the article provides an architectural assessment of the most recent type of genetic testing technology, single-nucleotide polymorphism (SNP, also known as whole genome) testing. SNP testing is based on the results of genomewide association studies that have linked pieces of an individual's genome sequence with particular traits, ancestry, and health conditions. Unlike genetic testing for Mendelian diseases (e.g., $B R C A$ ), SNP tests only pick up low levels of increased risk for a health condition (usually $<5 \%$ ). In recent years, a few 
for-profit companies have begun to offer the technology directly to clients outside the traditional medical setting. These new players, which join a growing number of direct-to-consumer genetic testing services, have provoked considerable attention and controversy. ${ }^{12-14}$ Some observers applaud the possibility of generating a large volume of genomic risk information that can inspire clients to adopt precautionary measures regarding their health and life. ${ }^{15,16}$ However, many worry that these services simply offer little "real" value or that they provide inconsistent information about an individual's risk of contracting a particular disease. ${ }^{5,17,18}$ This leads to additional concerns about whether the public has the tools to interpret the complicated and uncertain risk information that these tests generate, especially without the guidance of a trained physician. ${ }^{19-21}$

Will SNP testing technologies really offer these benefits? Are the concerns justified? Should we consider other positive or negative effects of this new technology? An assessment of the architectures of the SNP testing technologies currently available can help us to sharpen our assessment of their impact, even identifying consequences that we may not have previously considered. Also, by understanding the links between technological architectures and their impact, we can begin to think about how we might design future testing systems differently or whether we want to institute changes to the surrounding social system-e.g., educational campaigns for health care professionals and the public, regulatory frameworks - to maximize the benefits of currently available testing systems. This article focuses on SNP tests offered by two of the main commercial providers, 23andMe and Navigenics. Both are for-profit companies based in California. Conducting an architectural assessment in comparative perspective-particularly when focusing on technologies designed to perform essentially the same functions - can be particularly illuminating because it reveals the different options for design and assembly of system components and the impact of these architectural decisions. Because of space constraints, I focus here on how the architectural decisions made by these two companies influence (1) understandings of a test's utility and (2) the distribution of expertise among the participants - clients, health care professionals, and testing providers - in the medical system. The commentary concludes by exploring how architectural assessment can be useful for those who are eager to use genomics to improve the public's health and who are considering policy interventions to facilitate this process.

\section{SOCIOTECHNICAL ARCHITECTURES OF SNP TESTING}

At first glance, the SNP testing systems offered by 23 andMe and Navigenics seem quite similar. Both use a web interface, allow clients to order tests directly from them, analyze SNPs, and return results directly to the client. Thus, they seem to be similarly empowering, as they deliver genomic information that clients can use as they see fit. However, important differences arise when we analyze how each of these services perform their three main functions - providing access to their systems, analyzing biological material, and conveying test results. These differences, we will see, affect their societal impact: 23 andMe has built a test that presumes a well-informed client who can independently evaluate genomic information, whereas Navigenics' system preserves an important space for the expertise of scientists and health care professionals. These differences raise questions not only about how test reliability and utility should be assessed but also who should have the power to make these assessments.

\section{Accessing the test}

In many respects, clients of 23 andMe and Navigenics access testing in the same way. Both pay a fee online and receive a testing kit in the mail and must return the kit with a saliva sample and an informed consent form. Two differences, however, are significant. First, Navigenics' system is more expensive and offers less choice. 23andMe offers multiple testing packages, which range in price from $\$ 399$ to $\$ 499$. By contrast, Navigenics offers one service (\$999). More people are likely to use 23 andMe's service, because it is cheaper and because they can choose the type of analysis that will be useful to them. Indeed, 23andMe emphasizes "personal utility,"22,23 the idea that the client should have the power to determine the test's utility in the context of their needs. By contrast, Navigenics' clients pay more for a test that provides them access to more scientific and medical expertise-the company offers clients optional pretest counseling and only analyzes SNPs that have been reviewed and certified by its scientific, clinical, and policy advisory boards to be medically useful.

Second, the availability of a counselor's guidance (even by phone) makes Navigenics' system seem more recognizable, as clients are accustomed to undergoing tests with the support of a health care professional. However, this relationship between client and health care professional is not quite traditional because the testing company employs her. Navigenics' system may appeal to clients who appreciate the guidance, and even the gatekeeping, of scientific and medical experts, whereas 23 andMe may attract clients who are confident in their ability to interpret the genomic information generated.

\section{Analyzing the biological material}

Although 23 andMe and Navigenics both analyze SNPs, their testing platforms differ. 23andMe adds a SNP to its testing platform as soon as researchers have established its association with a particular trait or health condition, whereas Navigenics only adds a SNP once it has been approved by its own advisory boards. These boards make decisions according to the following criteria: (1) conditions the company deems medically relevant, (2) conditions that can be improved with early attention, and (3) relationships between SNPs and health conditions that have been established through "multiple, well-designed studies" to be "consistent, reliable, and significant." 24 Consequently, 23andMe currently analyzes four times as many SNPs as Navigenics. For example, although 23andMe tests SNPs related to schizophrenia, ${ }^{25}$ Navigenics does not because, it argues, "recent research into the genetic risk markers for this mental illness has yielded inconsistent results." 24 In sum, 23andMe offers to clients as many SNP tests as possible, whereas Navigenics emphasizes its gatekeeping apparatus.

These different strategies for analyzing the biological material define the expertise of system participants in different ways. 23andMe's approach suggests that clients have the ability, and should have the opportunity, to determine the reliability and utility of the results in the context of their own lives. By contrast, Navigenics trusts the scientists and medical professionals who sit on its advisory boards to make the same determinations and perform a gatekeeping role.

\section{Conveying the results}

Once 23andMe and Navigenics complete their analyses, both invite clients (by email) to review the results online. 23 and $\mathrm{Me}^{26}$ helps clients understand their results through "Clinical" and 
"Research" reports about each SNP-health condition association analyzed. Its scientific and medical advisors decide which type of report to issue based on the reliability of the research underlying the association. The company generates "Clinical" reports when researchers have identified clear associations ("supported by multiple, large, peer-reviewed studies") between a condition and a corresponding SNP and when the relationship is strong (when the riskiest possible combination of genotypes increases a person's odds of developing a condition at least threefold and when average lifetime risk of the disease is raised to at least $5 \%$ ). The company offers "Research" reports when a particular SNP has not been extensively studied or when an association does not dramatically influence a person's disease risk. In the case of SNPs related to schizophrenia, 23andMe issues a Research report.

By contrast, for each condition, Navigenics simply compares its client's risk to the general population's risk and informs her about the causes, symptoms, and preventative and treatment measures. Its scientific and medical experts have already judged the reliability and utility of the results by deciding which SNPs to analyze, and therefore, the company has no need to offer different kinds of reports. Once Navigenics has reported the test results, its clients have the freedom to decide how to use the information. In sum, although Navigenics' scientific and medical advisors do not make a SNP test available if they do not believe it is medically useful, their counterparts at 23 andMe are more likely to conduct the test but may offer the results in the context of a "Research" report - leaving the client to judge both the reliability and utility of the results.

Even after test results have been conveyed, both companies reinforce their distinct understandings of expertise. 23andMe has developed a social networking site $^{27}$ for clients, which privileges what some have called "experiential expertise"28,29; clients use their knowledge and experiences with 23andMe's system, and health care more broadly, to teach one another how to interpret risk information and respond to test results. Meanwhile, Navigenics' clients can consult again with the company's genetic counselors to help them interpret the results.

\section{THE IMPLICATIONS OF SOCIOTECHNICAL ARCHITECTURES}

Overall, although 23andMe provides as much information to its clients as possible for a low price, Navigenics offers a service that combines SNP analysis with health care services. These differences in business strategy lead to different sociotechnical architectures, which have different consequences. The 23 andMe's system emphasizes the expertise and autonomy of the client, allowing her to determine the reliability and utility of test results. Health care professionals play only a supplementary role. By giving clients the freedom to interpret genomic information independently, 23 andMe also takes no responsibility for the possibility of misinterpretation. Indeed, it is not possible for clients to misunderstand test results in this system, because 23 andMe trusts that clients interpret results in the manner that is best for them. However, the types of probabilistic information offered by SNP test results are extremely difficult to understand; even health care professionals have trouble interpreting genetic risk information. ${ }^{30,31}$ Thus, as a result of this system, medical specialists and public health professionals may have to adopt a more active role in their population-wide health promotion and education efforts, developing campaigns to help clients analyze the reliability of research in this area and to interpret the risk levels of the numerous test results. As they do this, they will have to become more sensitive to the utility of genomic information beyond the medical context.

The architecture of Navigenics' system, by contrast, envisions a user who appreciates scientific and medical expertise. Herein, scientific and medical experts set standards for the appropriateness, reliability, and utility of each test and also counsel clients. Although it may seem that Navigenics has recreated the traditional relationship between health care professionals and clients, it is important to remember that because the company ultimately provides both technical analysis and health advice, it controls both the information itself and how it is interpreted. This dual functionality raises conflict of interest concerns arising from the provision of health care services by a for-profit corporation who also generates the data. Also, clients may be less likely to seek analysis of test results from independent health professionals, believing that they have already received adequate advice. This approach could prove particularly problematic when outside health care professionals dispute Navigenics' choice of SNPs to analyze or its interpretation of risk information, because clients may not have much opportunity to hear these critiques. In dealing with this system, medical professionals and public health might actually have to perform a more difficult task, trying to educate the public as a whole with risk information that may contradict the analysis certified by Navigenics' scientific and medical experts.

\section{THE UTILITY OF ARCHITECTURAL ASSESSMENT}

Overall, architectural assessment can be useful in three ways. First, we can sharpen our understanding of a technology's impact. We have learned, for example, that discussions about the utility of SNP testing must take into account how different testing systems analyze biological material and convey test results. Is the public aware that different testing system architectures have different approaches to utility? How might this information influence their choice among services? Whose responsibility is it to make this information available? Second, we may uncover implications that have received comparatively less attention. Both SNP testing architectures examined in this study envision the distribution of expertise in the medical system in a nontraditional way. 23 andMe assumes the expertise of clients to interpret the reliability and utility of test results, whereas Navigenics takes on gatekeeping and counseling roles traditionally assigned to independent health care professionals. The architecture of a genetic testing technology could create new kinds of experts while diminishing the role and authority of traditional ones. Will these changes to our understandings of expertise be beneficial to health care? If not, what actions should we take?

Finally, we can consider changing a technology's architecture to maximize its benefits. Policymakers or professional associations, among others, could issue recommendations that establish a preference among the existing architectures. In the case of SNP testing, this approach would require serious conversations about the positive and negative dimensions of each provider's approach to, for example, test utility and the power and autonomy of clients, scientists, and health care professionals. Of course, choosing among existing architectures still limits the power of public health professionals and policymakers to shape the impact of these technologies. Alternatively, decision makers could envision an ideal architecture for SNP testing, which structures utility and the power of participants in the medical system in ways they believe to be best for the public. If they choose this option, they must work together with test providers, health care professionals, and clients to create an architecture that is simultaneously profitable, efficient, and ef- 
fective in providing the greatest possible benefit to the greatest number of people possible, a rather challenging task. Regardless, because policies must serve society's sustainable and longterm interests, policymakers must recognize that the impact of genomic technologies derive much from their sociotechnical architectures. The good news is that we can intervene in their design to maximize their benefits for the public's health and well-being.

\section{REFERENCES}

1. Carlson RJ. Preemptive public policy for genomics. J Health Polit Policy Law 2008;33:39-51.

2. Secretary's Advisory Committee on Genetics, Health, and Society. The integration of genetic technologies into health care and public health. SACGHS progress report and future directions. Bethesda, Maryland: Secretary's Advisory Committee on Genetics, Health, and Society, 2009.

3. Khoury MJ, McBride CM, Schully SD, et al. The Scientific Foundation for Personal Genomics: recommendations from a National Institutes of HealthCenters for Disease Control and Prevention multidisciplinary workshop. Genet Med 2009;11:559-567.

4. Rose N. Race, risk and medicine in the age of 'your own personal genome.' Biosocieties 2008;3:423-439.

5. Ng PC, Murray SS, Levy S, Venter JC. An agenda for personalized medicine. Nature 2009;461:724-726.

6. Robertson JA. The $\$ 1000$ genome: ethical and legal issues in whole genome sequencing of individuals. Am J Bioeth 2003;3:W-1F1.

7. Parthasarathy S. Building genetic medicine: breast cancer, technology, and the comparative politics of health care Cambridge, MA: MIT Press, 2007.

8. Bijker W, Hughes TP, Pinch T, editors. The social construction of technological systems: new directions in the sociology and history of technology. Cambridge, MA: MIT Press, 1989.

9. Wetmore JM. Redefining risks and redistributing responsibilities: building networks to increase automobile safety. Sci Technol Human Values 2004; 29:377-405.

10. Winner L. Do artifacts have politics? Daedalus 1980;109:121-136.

11. Bijker W. Of bicycles, bakelites, and bulbs: toward a theory of sociotechnical change. Cambridge, MA: MIT Press, 1997.

12. Shetty S. Home DNA test kits cause controversy. Lancet 2008;371:17391740 .
13. Berdick C. Your DNA on demand; new companies sell genetic readings direct to consumers, with no doctor involved. The Washington Post. January 26, 2010:HE01.

14. Pollack A. Firm brings gene tests to masses. The New York Times. January 29, 2010:B1.

15. Ledley F. A consumer charter for genomic services. Nat Biotechnol 2002; 20:767.

16. Wang G, Watts $C$. The role of genetics in the provision of essential public health services. Am J Public Health 2007;97:620-625.

17. Hunter DJ, Altschuler D, Rader DJ. From Darwin's finches to canaries in the coal mine-mining the genome for new biology. N Engl J Med 2008;358: $2760-2763$.

18. Bolnick DA, Fullwiley D, Duster T, et al. Genetics. The science and business of genetic ancestry testing. Science 2007;318:399-400.

19. Evans JP, Green RC. Direct to consumer genetic testing: avoiding a culture war. Genet Med 2009;11:568-569.

20. Gray S, Olopade OI. Direct-to-consumer marketing of genetic tests for cancer: buyer beware. J Clin Oncol 2003;21:3191-3193

21. McGuire AL, Burke W. An unwelcome side effect of direct-to-consumer personal genome testing: raiding the medical commons. JAMA 2008;300: 2669-2671.

22. Grosse SD, McBride CM, Evans JP, Khoury MJ. Personal utility and genomic information: look before you leap. Genet Med 2009;11:575-576.

23. Foster MW, Mulvihill JJ, Sharp RR. Evaluating the utility of personal genomic information. Genet Med 2009;11:570-574.

24. Navigenics. Conditions we cover. Available at: http://www.navigenics.com/ visitor/what_we_offer/conditions_we_cover/. Accessed November 3, 2009.

25. 23andMe.Schizophrenia - samplereport. Availableat:https://www.23andme com/health/Schizophrenia/. Accessed November 3, 2009.

26. 23andMe. Health and traits: complete list. Available at: https://www.23andme. com/health/all/. Accessed November 3, 2009.

27. 23andMe. Sharing and community. Available at: https://www.23andme.com/ community/. Accessed November 3, 2009.

28. Epstein S. Impure science: AIDS, activism, and the politics of knowledge. Berkeley, CA: University of California Press, 1996.

29. Caron-Flinterman JF, Broerse JE, Bunders JF. The experiential knowledge of patients: a new resource for biomedical research? Soc Sci Med 2005;60: 2575-2584.

30. Rich EC, Burke W, Heaton CJ, et al. Reconsidering the family history in primary care. J Gen Intern Med 2004;19:273-280.

31. Emery J, Hayflick S. The challenge of integrating genetic medicine into primary care. BMJ 2001;322:1027-1030. 